\title{
Dek504 Encodes a Mitochondrion-Targeted E+-Type Pentatricopeptide Repeat Protein Essential for RNA Editing and Seed Development in Maize
}

\author{
Zheyuan Wang ${ }^{1,2}$, Weiwei Chen ${ }^{1,2}$, Song Zhang ${ }^{1,2}$, Jiawen Lu ${ }^{1,2}$, Rongrong Chen ${ }^{2}$, Junjie Fu ${ }^{2}$, Riliang Gu ${ }^{1} \mathbb{D}$, \\ Guoying Wang ${ }^{2}$, Jianhua Wang ${ }^{1, *}$ and Yu Cui ${ }^{2, *}$
}

1 Beijing Innovation Center for Crop Seed Technology, Ministry of Agriculture and Rural Affairs, Key Laboratory of Crop Heterosis Utilization, Ministry of Education, College of Agronomyand Biotechnology, China Agricultural University, Beijing 100193, China; wangzheyuan@cau.edu.cn (Z.W.); 15501062618@163.com (W.C.); zhangsong0115@163.com (S.Z.); lujiawen@cau.edu.cn (J.L.); rilianggu@cau.edu.cn (R.G.)

2 Institute of Crop Sciences, Chinese Academy of Agricultural Sciences, Beijing 100081, China; 670702crr@163.com (R.C.); fujunjie@caas.cn (J.F.); wangguoying@caas.cn (G.W.)

* Correspondence: wangjh63@cau.edu.cn (J.W.); cuiyu@caas.cn (Y.C.)

check for updates

Citation: Wang, Z.; Chen, W.; Zhang, S.; Lu, J.; Chen, R.; Fu, J.; Gu, R.; Wang, G.; Wang, J.; Cui, Y. Dek504 Encodes a Mitochondrion-Targeted E+-Type Pentatricopeptide Repeat Protein Essential for RNA Editing and Seed Development in Maize. Int. J. Mol. Sci. 2022, 23, 2513. https:// doi.org/10.3390/ijms23052513

Academic Editor: Lars Matthias Voll

Received: 12 January 2022

Accepted: 22 February 2022

Published: 24 February 2022

Publisher's Note: MDPI stays neutral with regard to jurisdictional claims in published maps and institutional affiliations.

Copyright: (c) 2022 by the authors. Licensee MDPI, Basel, Switzerland. This article is an open access article distributed under the terms and conditions of the Creative Commons Attribution (CC BY) license (https:// creativecommons.org/licenses/by/ $4.0 /)$.

\begin{abstract}
In flowering plants, RNA editing is a post-transcriptional process that selectively deaminates cytidines $(\mathrm{C})$ to uridines $(\mathrm{U})$ in organellar transcripts. Pentatricopeptide repeat $(\mathrm{PPR})$ proteins have been identified as site-specific recognition factors for RNA editing. Here, we report the mapbased cloning and molecular characterization of the defective kernel mutant dek504 in maize. Loss of Dek504 function leads to delayed embryogenesis and endosperm development, which produce small and collapsed kernels. Dek504 encodes an E+-type PPR protein targeted to the mitochondria, which is required for RNA editing of mitochondrial NADH dehydrogenase 3 at the nad3-317 and nad3-44 sites. Biochemical analysis of mitochondrial protein complexes revealed a significant reduction in the mitochondrial NADH dehydrogenase complex I activity, indicating that the alteration of the amino acid sequence at nad3-44 and nad3-317 through RNA editing is essential for NAD3 function. Moreover, the amino acids are highly conserved in monocots and eudicots, whereas the events of C-to-U editing are not conserved in flowering plants. Thus, our results indicate that Dek504 is essential for RNA editing of nad3, which is critical for NAD3 function, mitochondrial complex I stability, and seed development in maize.
\end{abstract}

Keywords: E+-type; kernel development; maize; mitochondria; NAD3; PPR; RNA editing

\section{Introduction}

RNA editing is a post-transcriptional modification occurring in vascular plant organelles through a deamination reaction at a specific nucleotide cytidine $(\mathrm{C})$, which is converted to uridine (U) [1,2]. The major consequence of RNA editing is that the amino acid (aa) sequence differs from that encoded from the genome sequence. Typically, the amino acids encoded by edited mRNAs are more conserved than the genome-encoded ones [3]. Currently, RNA editing is viewed as a remedial DNA mutation at the RNA level to restore the evolutionarily conserved amino acids [3-5].

In angiosperms, most organelle transcripts harbor hundreds of editing sites. However, when plant mitochondrial RNA editing was discovered over 30 years ago, little was known regarding the molecular apparatus involved in identifying the correct $C$ editing targets $[6,7]$. In 2005, Chlororespiratory Reduction 4 (CRR4), which possesses a pentatricopeptide repeat (PPR) motif, was found to be essential for the RNA editing of the chloroplast $n d h D$ gene [8]. Since then, members of the PPR protein family have been identified as RNA editing factors required for site-specific C-to-U conversions in chloroplasts or mitochondria [9-13]. 
PPR proteins are present in all eukaryotes and constitute one of the largest protein families, with over 400 members in most species [14,15]. Based on the structure of their PPR motifs, these proteins are divided into two major subclasses: (i) P-PPR proteins, which only harbor canonical P motifs of 35 amino acids, and (ii) PLS-PPR proteins, which harbor the canonical P motif, a long (L, 35-36 aa) motif, and a short (S, 31-32 aa) motif, forming tandemly repeated P-L-S triplets [16,17]. The majority of the PLS-PPR proteins contain additional C-terminal E, E+, and DYW domains [16]. P-PPR proteins are mainly involved in various aspects of organelle RNA processing, whereas PLS-PPR proteins are almost exclusively related to C-to-U conversion [18].

In addition to PPR proteins, C-to-U RNA editing involves proteins from diverse families, including multiple organelle RNA editing factors/RNA-editing factor interacting proteins (MORFs/RIPs) [19,20], organelle RNA recognition motif-containing proteins (ORRMs) [21], protoporphyrinogen IX oxidase 1 (PPO1) [22], and organelle zinc finger proteins (OZ1) [23]. Accumulating evidence indicates that PPR proteins regulate C-toU RNA editing in plant organelles via forming an editing complex with other editing factors $[9,19,24-29]$.

The maize NB mitochondrial genome harbors 493 editing sites in 58 mitochondrial genes [28], with 399 sites in 22 genes encoding the subunits of electron transport chain (ETC) complexes (I-V) and cytochrome c biogenesis proteins (e.g., nad1, nad2, nad3, nad4, nad4L, nad5, nad6, nad7, nad9, cob, cox1, cox2, cox3, atp1, atp4, atp6, atp8, ccmB, ccmC, ccmFC, and $c c m F N)$ [30]. To date, 19 PLS-PPR proteins have been shown to be essential for the C-to-U RNA editing of mitochondrial transcripts in maize (Table S1). Of these, 5 proteins, namely PPR27, DEK53, DEK55, EMP5, and EMP21, are involved in editing at multiple sites, and the remaining 14 are involved in the editing at one or a few specific sites [9,26-29]. The loss-of-function of these PPR proteins abolishes editing or reduces editing efficiency, ultimately affecting the assembly and activity of the related mitochondrial complexes. Simultaneously, the alternative oxidase (AOX) pathway is activated, indicating defective electron transport in the cytochrome c pathway [31,32]. The mitochondria serve as the energy supply and redox regulation center in cells [33], which convert the chemical energy stored in carbon substrates to adenosine triphosphate (ATP) via ETC [33-35]. Typically, the impairment of mitochondrial function delays embryonic and endosperm development and arrests basal endosperm transfer layer (BETL) development, ultimately producing mutant phenotypes with empty pericarps (emp) and defective or small kernels (dek or smk, respectively) $[9,29,36,37]$.

PPR proteins play pivotal roles in RNA editing to maintain mitochondrial function during embryonic and endosperm development. In the present study, a new maize dek mutant, dek504, exhibiting a small and delayed kernel development phenotype, was characterized. DEK504 encodes a mitochondrion-targeted E+-type PPR protein that is involved in C-to-U editing at specific positions of NADH dehydrogenase gene 3 (nad3-44 and nad3-317). Defects at these editing sites impede the assembly and stability of mitochondrial complex I, thus impairing the structure and function of mitochondria and arresting the development of embryos and endosperm in maize.

\section{Results}

\subsection{Genetic and Phenotypic Characterization of the dek504-ref Mutant}

A spontaneous mutant exhibiting a defective kernel phenotype was isolated from an inbred line $14 d 3147$ and named dek504-ref. The segregation ratio of normal to defective kernels on the self-crossed ears of dek504-ref heterozygote was consistent with the Mendelian separation ratio of 3:1 $\left(\chi^{2}=0.530<\chi^{2}{ }_{0.05}=3.84\right.$; Figure 1a), indicating that dek504-ref is a monogenic recessive mutation. This result was confirmed in the $\mathrm{F}_{2}$ populations generated from the crosses of dek504-ref/+ with B73, Mo17, and Zheng58 inbred lines (Supplementary Figure S1a-c). 


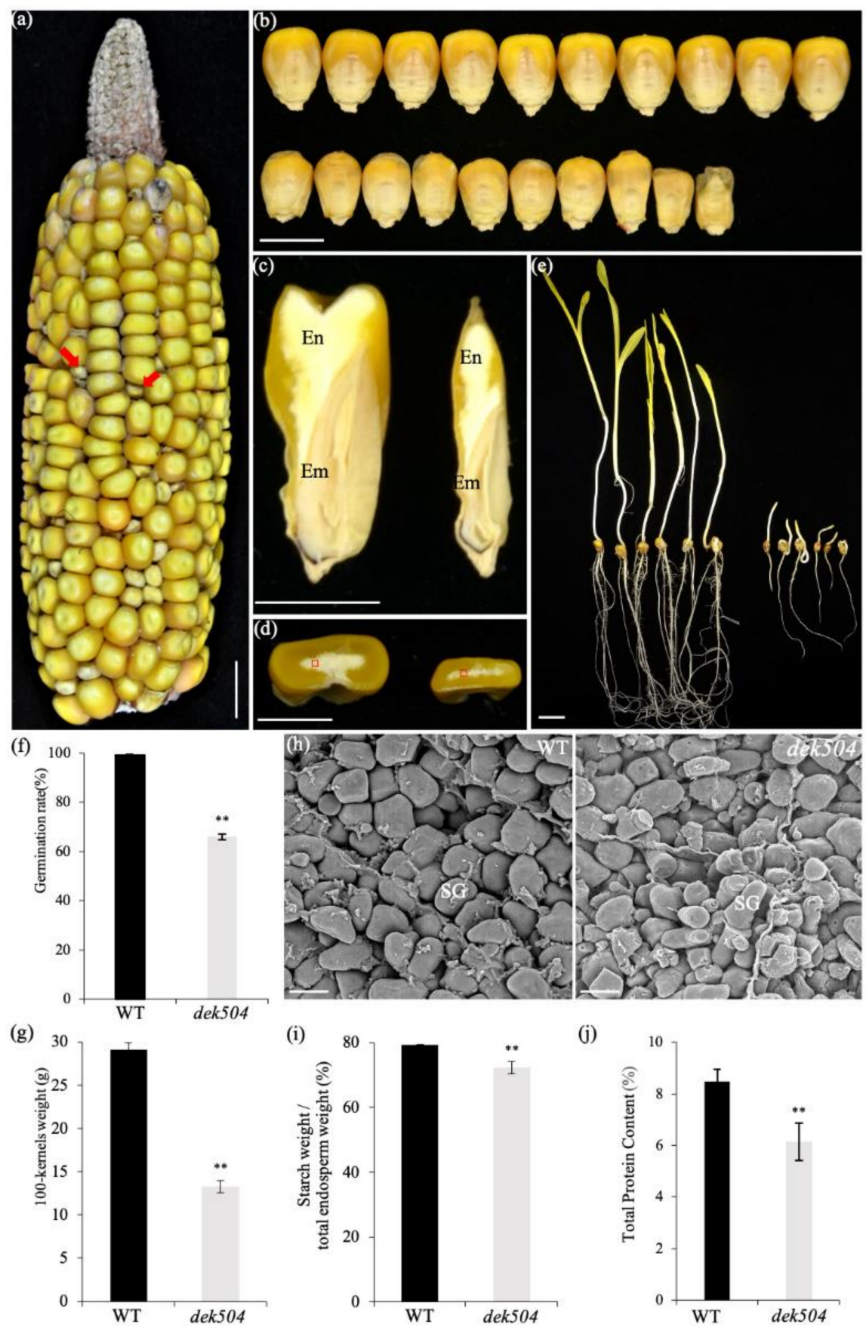

Figure 1. Phenotypic characteristics of the maize dek504-ref mutant. (a) Mature self-pollinated ear of dek504-ref heterozygous plant; red arrow indicates the dek504 kernel. Bar $=1 \mathrm{~cm}$. (b) Front view of wildtype (WT, up) and dek504-ref (down) kernels. Bar $=1 \mathrm{~cm}$. (c,d) Longitudinal (c) and transverse (d) sections of WT (left) and dek504-ref (right) kernels. Bar $=0.5 \mathrm{~cm}$. (e,f) Germination test (e) and rate (f) of WT and dek504-ref seedlings at 7 days after germination (DAG). Bar $=2 \mathrm{~cm}$. (g) Comparison of the 100-kernel weight of randomly selected mature WT and dek504-ref plants. (h) Scanning electron micrographs of WT and dek504-ref mature endosperm. SG, starch granule. Bar $=10 \mu \mathrm{m}(\mathbf{i}, \mathbf{j})$ Starch (i) and total protein (j) content of WT and dek504-ref endosperm at the mature stage. Values indicate means $\pm \operatorname{SD}(n=3 ; * * p<0.01$, Student's $t$-test $)$.

Contrary to the wildtype (WT) maize, dek504 developed small kernels and appeared collapsed at maturity, with dramatic size and weight reduction (Figure 1b). The 100-kernel weight of dek504 was $45.7 \%$ of the weight of WT (Figure 1g). In longitudinal and transverse sections, dek504 kernels showed distinctly wrinkled embryo and endosperm structures (Figure 1c,d). Approximately 65\% of the dek504 kernels germinated, although the germinated seedlings showed retarded growth (Figure 1f). While the WT seedlings formed a primary root and several seminal roots after 7 days of germination, dek504 seedlings only formed a primary root. The length of the primary roots and shoots was significantly shorter in dek504 seedlings than in WT seedlings (Figure 1e). Moreover, some of the germinated dek504 seedlings completed the entire growth period and produced small seeds with a visible collapsed seed coat (Supplementary Figure S1d,e).

Scanning electron microscopy (SEM) revealed that the starch granules (SGs) in WT internal endosperm cells were smooth and tightly packed. However, SGs in dek504 en- 
dosperm cells were loosely and irregularly packed and exhibited many holes on the surface (Figure 1h). In mature kernels, the total starch and protein contents in dek504 endosperm were, respectively, 8.7\% and 27.5\% lower than those in WT endosperm (Figure 1i,j). Overall, the dek504 mutation delayed plant growth and seed development as well as lowered seed nutrient reservoir accumulation.

The developing kernels of dek504 and WT were observed at 12, 15, and 18 days after pollination (DAP). The dek504 kernels could be distinguished from the WT kernels as early as 12 DAP, characterized by their small size and translucent appearance with white top (Figure 2a). Paraffin-embedded sections showed that the WT embryos developed visible scutellum, coleoptile, leaf primordium (LP), and shoot apical meristem (SAM) and reached the L2 (second leaf primordia) stage at 12 DAP (Figure 2b); whereas dek504 embryos arrested and remained at the transition stage, without visible differentiation in LP and SAM. The WT embryo developed 3-4 leaf primordia and complete structures with clearly visible SAM and root apical meristem (RAM) at 15 and 18 DAP, reaching late embryogenesis stage (Figure 2c-e). By contrast, the dek2849 embryos reached the L1 stage with one leaf primordium and SAM but without RAM at 15 DAP. At 18 DAP, the mutant embryos remained at the L1 stage and showed no significant sign of RAM development (Figure 2c-e). In addition, compared with WT kernels, which had starchfilled endosperms, the dek504 kernels had small and underdeveloped endosperms, with a gap observed between the endosperm and seed coat at 12 DAP (Figure 2b). The dek504 endosperm development was slower than that of the WT at 15 and 18 DAP (Figure 2c,d). The WT kernels showed extensive cell wall ingrowth in the basal endosperm transfer layer (BETL) cells at 15 DAP, whereas dek504 kernels comprised small cells and showed little cell wall ingrowth in BETL (Figure 2f). Overall, the dek504-ref mutation affected both embryonic and endosperm development.
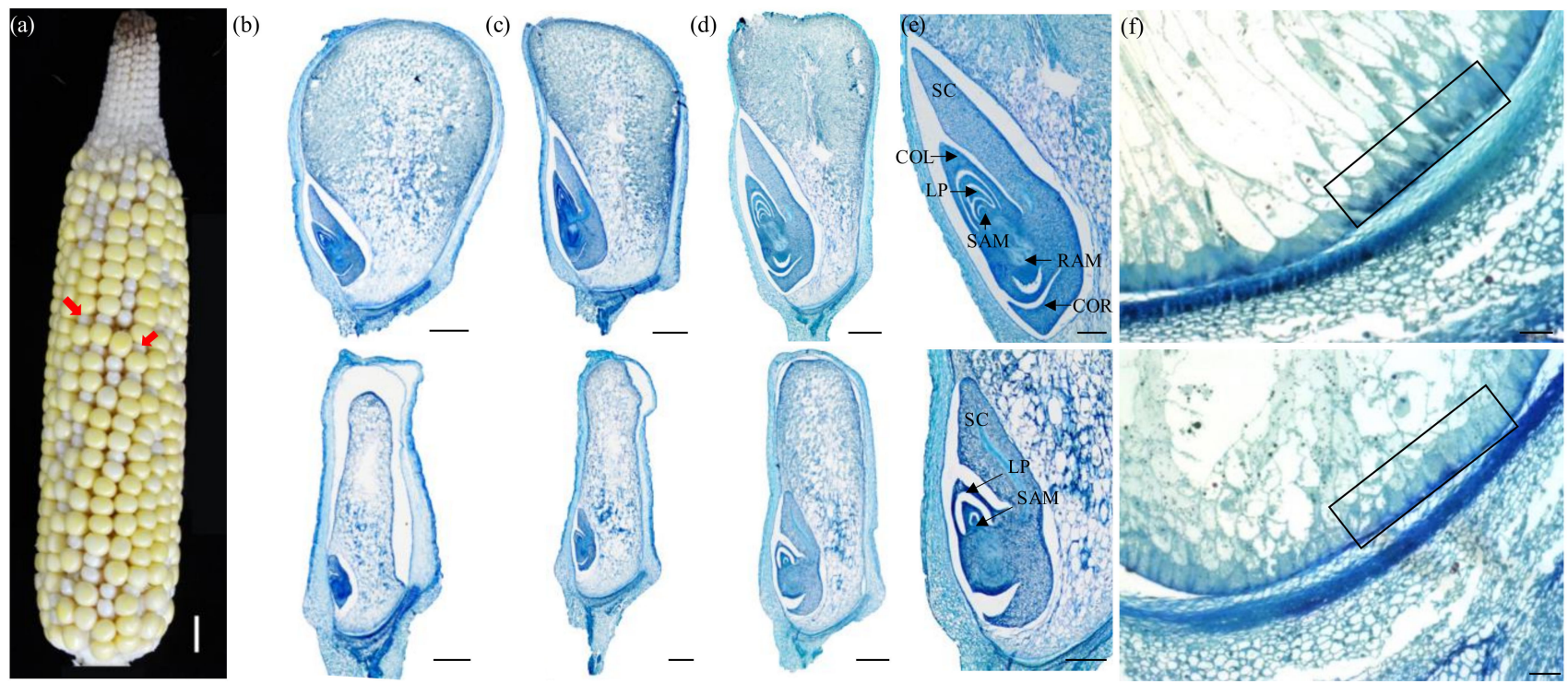

Figure 2. Comparison of developing kernels between wildtype (WT) and dek504-ref maize. (a) Self-pollinated dek504-ref heterozygote ears at 12 days after pollination (DAP). Bar $=1 \mathrm{~cm}$. (b-d) Histological analysis of WT (above) and dek504 (bottom) kernel at 12 (b), 15 (c), and 18 DAP (d). Bar $=1 \mathrm{~cm}$. (e) Magnified image of Figure 2d. SC, scutellum; COL, coleoptile; LP, leaf primordium; SAM, shoot apical meristem; RAM, root apical meristem; COR, coleorhiza. Bar $=1 \mathrm{~mm}$. (f) Comparison of the basal endosperm transfer layer (BETL) between WT and dek504 at 15 DAP. Bar $=50 \mu \mathrm{m}$.

\subsection{Positional Cloning of dek504}

The dek504-ref heterozygotes were crossed with the B73 inbred line to generate an $\mathrm{F}_{2}$ population for the map-based cloning of dek504. Homozygous mutant and WT kernels from segregated F2 ears at 15 DAP were used for bulked segregant RNA-Seq (BSR- 
Seq), which initially mapped the dek504 gene to a $6.7 \mathrm{Mb}$ interval on chromosome 7 (Supplementary Figure S2). After characterizing 6192 homozygous mutant kernels using seven markers within this $6.7 \mathrm{Mb}$ interval (Table S2), the mapping region was narrowed to a $60.3 \mathrm{~Kb}$ region containing four putative protein-coding genes, namely G1-Zm00001d022392, G2-Zm00001d022393, G3-Zm00001d022394, and G4-Zm00001d022395, according to the B73 reference genome (AGPv4) (Figure 3a). Genomic sequencing revealed the same sequence of G1 and G2 open reading fragments (ORFs) between WT and dek504 but revealed a $6.9 \mathrm{~Kb}$ deletion in the dek504 genome from $176,672,153$ to $176,679,118 \mathrm{bp}$. This deletion led to the complete loss of the G4 ORF and partial loss of the G3 ORF (Figure 3a-c). Reverse transcription-polymerase chain reaction (RT-PCR) failed to amplify the transcripts of G3 and G4 ORFs, further confirming the presence of this deletion.

(a)

(b)

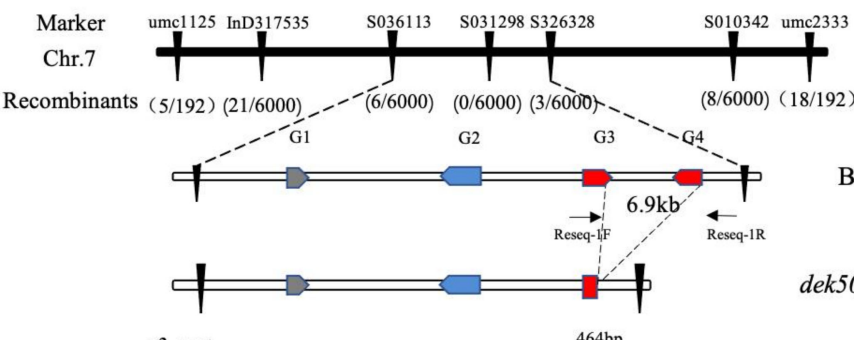

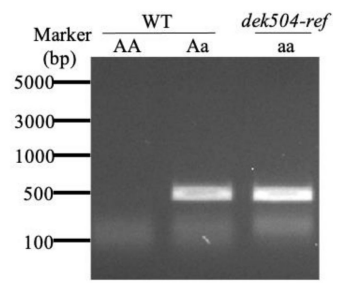

(d)
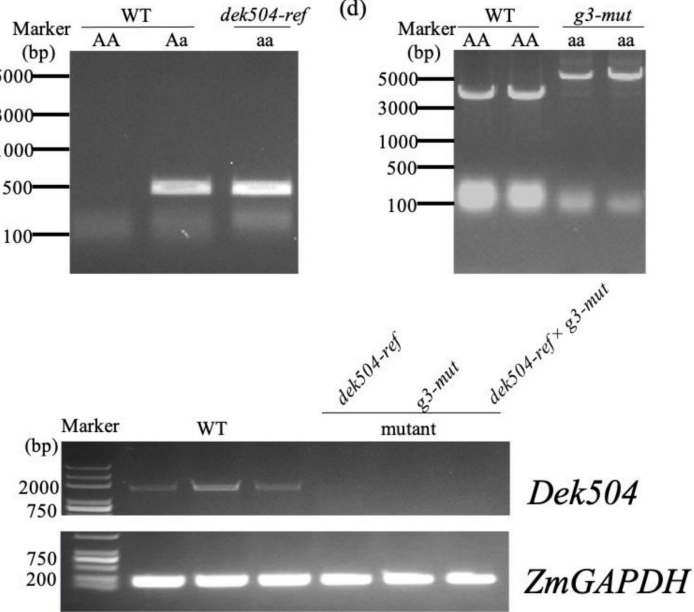

(f)

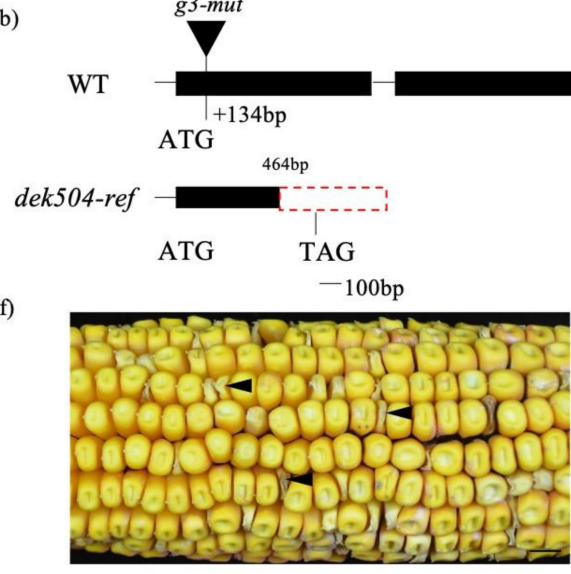

dek504-refl $+\times$ dek504-reff +

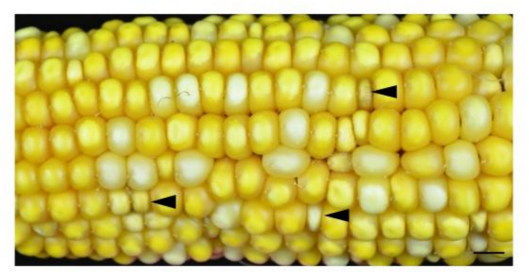

g3-mut/+ Xg3-mut/+

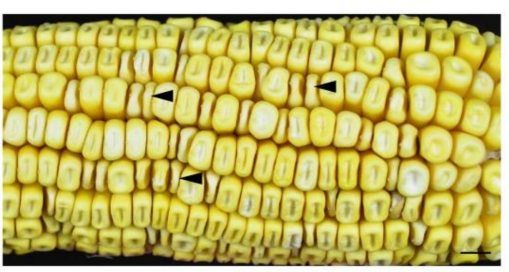

dek504-ref/+× g3-mut/+

Figure 3. Cloning and identification of dek504. (a) Fine mapping of the dek504 locus. The dek504 locus was mapped to a $70 \mathrm{~kb}$ region on chromosome 7, harboring four putative genes. (b) Gene structure and mutation site of Dek504. (c,d) Genotype analysis of wildtype (WT), dek504-ref (c), and g3-mut (d) kernels. (e) Full-length open reading frame (ORF) amplification of Dek504 expression in WT and dek504 mutant alleles using RT-PCR. Expression was normalized against ZmGAPDH. (f) Heterozygous dek504-ref and g3-mut were used in the allelism test of Dek504. Black arrow indicates the mutant kernel. Bar $=1 \mathrm{~cm}$.

To determine the causal gene for dek504, the G3 (g3-mut) and G4 (g4-ems) mutants were characterized. g4-ems was an EMS mutant, containing a single-nucleotide substitution (C to T) at $396 \mathrm{bp}$ of the G4 ORF, resulting in a premature stop codon and a truncated protein (Supplementary Figure S3a). g3-mut was a spontaneous mutant, carrying a 3017 bp Gypsy31-ZM_LTR insertion at 134 bp of the G3 ORF (Figure 3b,d). The heterozygous g4-ems and g3-mut mutants were crossed with the heterozygous dek504-ref mutant for allelic testing, which revealed that the kernels generated from the cross between dek504-ref and g3-mut exhibited a 3:1 segregation ratio of the WT and dek phenotypes $\left(681: 211, \chi^{2}=2.509<\chi^{2}{ }_{0.05}=3.84\right.$; Figure 3f); however, all kernels generated from the cross between dek504-ref and g4-ems exhibited the WT phenotype (Supplementary Figure S3b). Therefore, G3 (Zm00001d022394) but not G4 is the causative gene of dek504. 


\subsection{Dek504 Encodes a Mitochondrion-Targeted E+-Type PPR Protein}

RACE experiments revealed that the Dek504 ORF in WT was $1830 \mathrm{bp}$. However, in the dek504 mutant, the genome deletion led to the loss of ORF sequence from +465 to $+1830 \mathrm{bp}$, and the addition of a $157 \mathrm{bp}$ transcript from the flanking genome sequence resulted in a mutant ORF of $621 \mathrm{bp}$ (Supplementary Figure S4). DEK504 was predicted to encode a $66 \mathrm{kD}$ PPR protein. Motif prediction analysis using PLANTPPR (https: / ppr.plantenergy. uwa.edu.au/, accessed on 27 April 2019) and TPRpred (http://tprpred.tuebingen.mpg.de/ tprpred, accessed on 27 April 2019) [17,38] revealed that DEK504 contained 12 PPR motifs, one E1/E2 domain, and one E+ domain; therefore, the protein was classified as an E+-type PLS-PPR protein (Figure 4a). The truncated DEK504 protein in the dek504 mutant lacked the last 10 PPR motifs, the E1/E2 domain, and the E+ domain (Figure 4a).
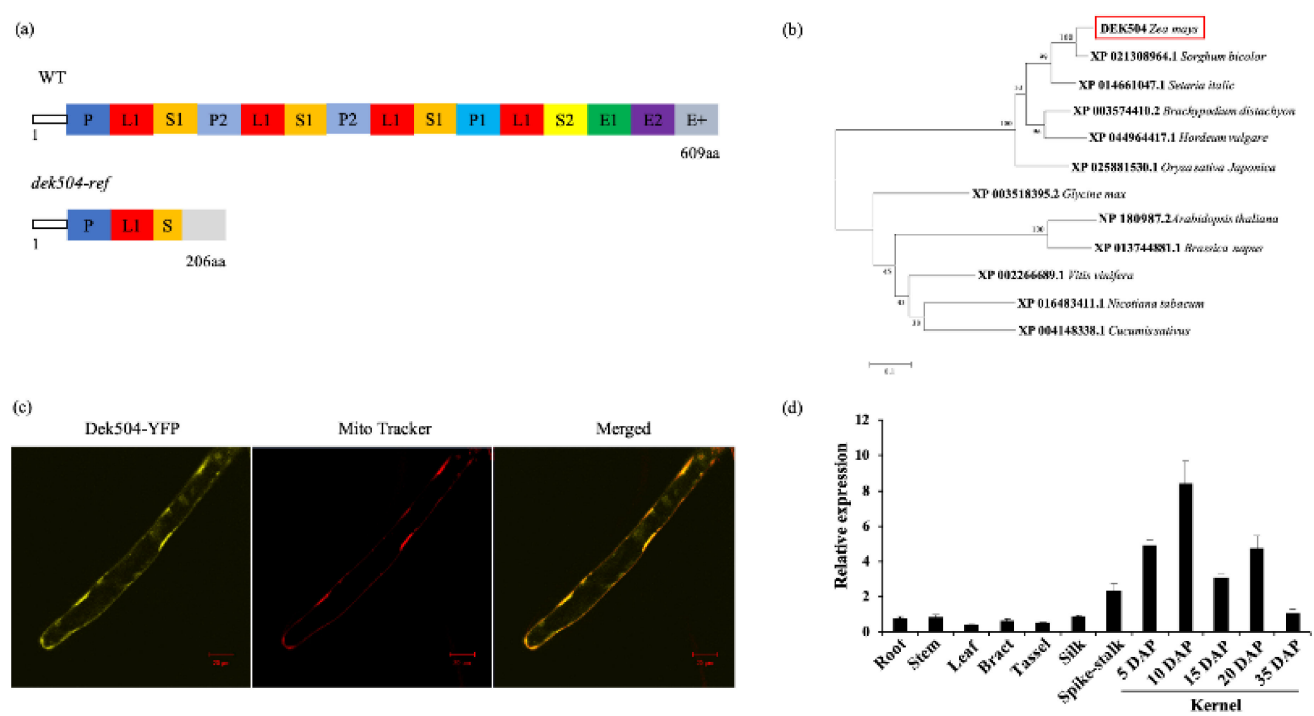

Figure 4. Maize Dek504 encodes a mitochondrion-targeted E+-type pentatricopeptide repeat (PPR) protein. (a) Schematic diagram of the maize DEK504 protein harboring 15 PPR domains (P, L, and S domains) and C-terminal E1, E2, and E+ motifs as well as of dek504-ref with only one PPR domain. (b) Phylogenetic relationships of DEK504 and its homologs in Cucumis sativus, Nicotiana tabacum, Vitis vinifera, Glycine max, Brassica napus, Arabidopsis thaliana, Oryza sativa Japonica, Hordeum vulgare, Brachypodium distachyon, Setaria italica, and Sorghum bicolor. Numbers at the nodes represent the percentage of 1000 bootstraps. (c) Subcellular localization of the DEK504 protein in Arabidopsis roots. The yellow auto-fluorescent signal was generated by DEK504-YFP, and the red fluorescence of the mitochondria was detected using MitoTracker. Bar $=20 \mu \mathrm{m}$. (d) Expression profiles of Dek504 in various organs and maize kernels at different developmental stages. $\mathrm{ZmGAPDH}$ was used as the reference gene. Values represent the means of three technical replicates, and the error bars represent SE.

Phylogenetic analysis based on the protein sequence of 12 DEK504 homologs from 12 species revealed that these proteins diverged into two separate clades of monocots and eudicots (Figure 4b). DEK504 showed a higher similarity to monocot homologs $(75.54 \sim 91.3 \%)$ than to eudicots (42.49 48.82\%), with the highest similarity (91.3\%) with the Sorghum bicolor homolog (Figure $4 \mathrm{~b}$ ). The detailed sequence alignment revealed highly conserved P/L/S/E domains among monocot DEK504 homologs (Supplementary Figure S5).

TargetP (http:/ /www.cbs.dtu.dk/services/TargetP/, accessed on 4 May 2019) and Predotar (http:/ / urgi.versailles.inra.fr/predotar/predotar.html, accessed on 4 May 2019) predicted that DEK504 was localized in the mitochondria. Next, the Dek504 ORF was fused to the gene encoding an enhanced yellow fluorescent protein (eYFP) driven by the CaMV $35 S$ promoter and transformed into Arabidopsis. The fluorescent signal of DEK504-eYFP overlapped with the red fluorescence of the mitochondrial localization marker MitoTracker pBIN20-MT-YB [39], indicating that DEK504 was targeted to the mitochondria (Figure 4c). 
Quantitative RT-PCR (qRT-PCR) revealed that Dek504 was constitutively expressed in all tested organs, including roots, stems, leaves, bracts, tassels, silk, and spike stalk, with relatively higher expression in kernels (Figure 4d). During kernel development, Dek504 expression was detected at 5 DAP; its expression gradually increased thereafter, peaking at 10 DAP, and then gradually decreased at the later stages (Figure $4 \mathrm{~d}$ ).

\subsection{DEK504 Is Required for the C-to-U Editing of Mitochondrial nad3 Transcript}

A previous study showed that PPR-E+ proteins are involved in the C-to-U editing of mitochondrial RNA [18]. Therefore, the editing sites of DEK504 were explored using the RNA-Seq of dek504 mutant and WT kernels of 15 DAP. The mutation did not significantly affect the editing sites of mitochondrial transcripts. However, editing at nad3-44 (from 80\% to $40 \%$ ) and nad3-317 (from $94 \%$ to $<1 \%$ ) was dramatically decreased in dek504 kernels. The DNA nucleotides of nad3-44 and nad3-317 sites were the same between dek504 and WT kernels, excluding the possibility that polymorphisms (SNPs) affect editing efficiency. The RNA editing sites in WT and dek504 were confirmed by RT-PCR, which demonstrated a reduction in editing from $70 \%$ to $8 \%$ at nad3-44 and from $75 \%$ to $0 \%$ on nad3-317 in the dek504 kernel (Figure 5a). According to the codes defined by the combinatorial residues at positions 6 and $1[15,40]$, the identity of the combinatorial codes of PPR repeats well matched the recognition sites around the RNA editing sites nad3-44 and nad3-317 (Figure 5b). Therefore, the DEK504 protein is necessary for RNA editing at nad3-44 and nad3-317 in maize.

(a)

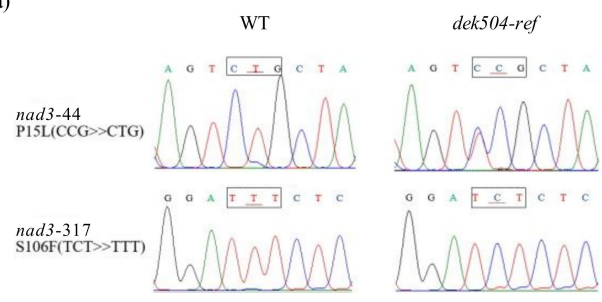

(c)

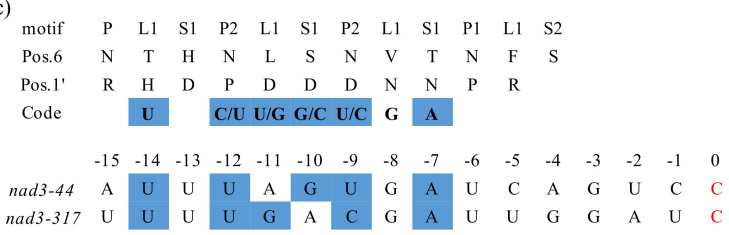

(b)

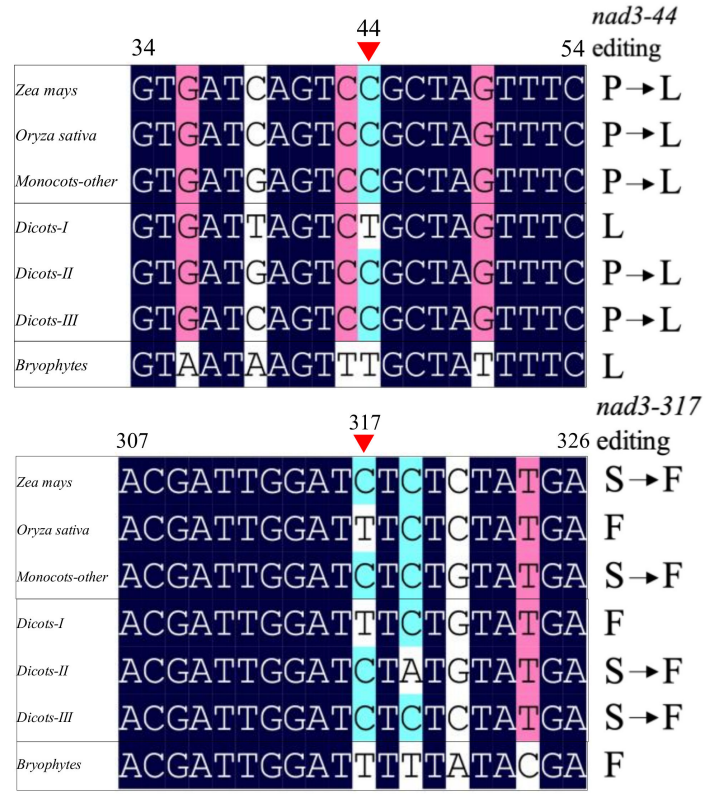

Figure 5. DEK504 is required for nad3-44 and nad3-317 C-to-U editing in maize mitochondria. (a) Analysis of RNA editing in nad3 transcripts of wildtype (WT) and dek504-ref kernels. The editing sites are indicated by the red lines. Codons harboring the edited nucleotides and coded amino acids are shown. (b) Alignment of amino acid residues at positions 6 and $1^{\prime}$ in each PPR motif of DEK504 with a -1 to -15 bp upstream sequence of these four defective editing sites. Nucleotides matching the recognition code of DEK504 in nad3 are shown in blue. The edited C residues in nad3 are shown in red. (c) Alignment of the neighboring gDNA sequences of nad3. The gDNA and cDNA sequences were obtained from the NCBI database. Monocots included Zea luxurians, Hordeum vulgare, Sorghum bicolor, Triticum aestivum, Bambusa oldhamii, Avena sativa, Phoenix dactylifera, Stipa capillata, Allium cepa, and Coix lacryma-jobi. Dicots-I included Arabidopsis thaliana, Brassica juncea, Brassica rapa, Brassica napus, and Boechera stricta. Dicots-II included Gossypium trilobum, Lagerstroemia indica, Bombax ceiba, Fagus sylvatica, Manihot esculenta, Heuchera parviflora, Malania oleifera, and Damnacanthus indicus. Dicots-III included Cannabis sativa, Panax vietnamensis, Panax notoginseng, and Tamarindus indica. Red triangles indicate the positions affected by nad3-44 and nad3-317. 
Deficient editing at nad3-44 and nad3-317 in the dek504 mutant led to a Leu-to-Pro conversion at NAD3-15 and a Phe-to-Ser change at NAD3-106 (Figure 5a). gDNA sequence alignment showed that both editing sites encoded " $\mathrm{C}$ " in monocots, with the exception of " $\mathrm{T}$ " at nad3-317 in rice; however, both editing sites encoded " $\mathrm{T}$ " in lower plants, and " $\mathrm{C}$ " or " $\mathrm{T}$ " remained in eudicots. cDNA sequence alignment of NAD3 orthologs revealed that these two amino acids were conserved in bryophytes, dicots, and monocots (Figure 5c), implying their significance for the functional integrity of NAD3 proteins.

\subsection{Dek504 Mutation Affects Mitochondrial Complex I Assembly and NADH Dehydrogenase Activity}

Previous studies have reported that editing defects in mitochondrial transcripts affect the activity of mitochondrial respiratory complexes and lead to mitochondrial dysfunction [41-43]. To further investigate whether changes in nad3 editing efficiency affect mitochondrial complex I formation and function, mitochondrial proteins were isolated from dek504-ref and WT kernels and analyzed by blue native polyacrylamide gel electrophoresis (BN-PAGE). There was a slight decrease in mitochondrial complex I and super complex $\mathrm{I}+\mathrm{III}_{2}$ formation, but a slight increase in complex III and V formation in dek504-ref compared with that in WT (Figure 6a). The NADH dehydrogenase activity of complex I was quantitatively evaluated using ImageJ (https: / /imagej.nih.gov/ij/ , 1.6.0_24, accessed on 4 October 2019) [44], which revealed a 43\% decrease in dek504-ref (Figure 6b). Therefore, the reduction in editing efficiency at nad3 impeded complex I assembly and activity in dek504.

(a)

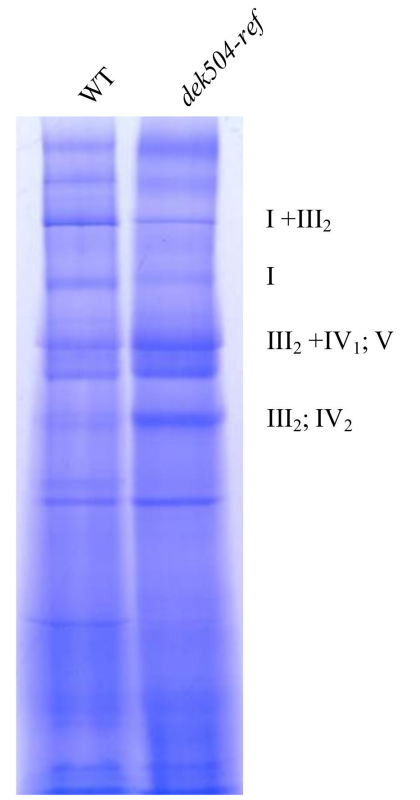

(b)

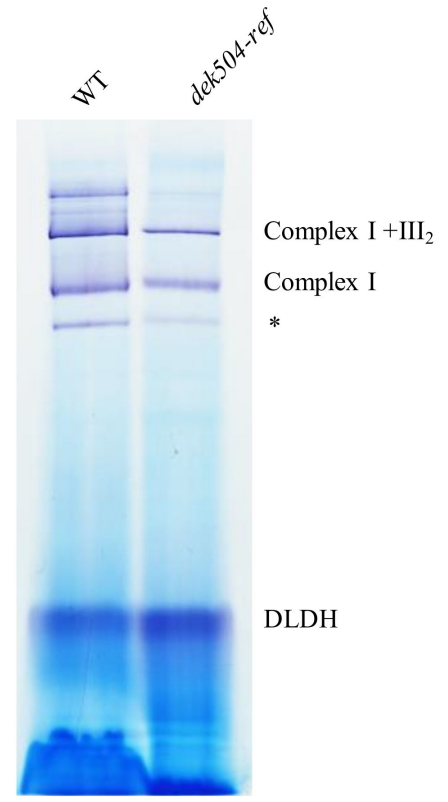

(c)
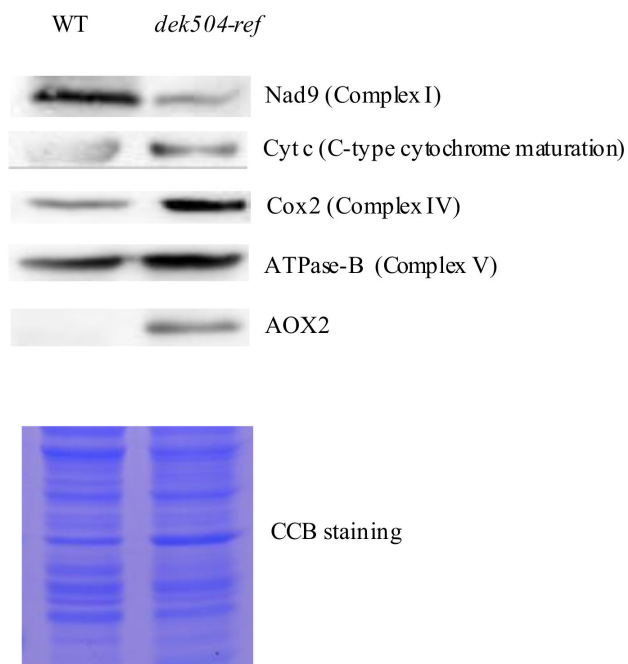

Figure 6. dek504 mutation affected the assembly and activity of mitochondrial complex I. (a) BNPAGE of the mitochondrial complexes. The positions of super complex $\mathrm{I}+\mathrm{III}_{2}$, complex I, complex III, and complex IV are indicated. (b) In-gel NADH dehydrogenase activity assay of complex I. The positions of super complex $\mathrm{I}+\mathrm{III}_{2}$ and complex I are indicated. DLDH was used as the loading control with the asterisk $\left(^{*}\right)$ representing the partially assembled complex I. (c) Western blotting with antibodies against NAD9, Cytc, Cox2, ATPase-B, and AOX.

To assess the impact of defective RNA editing at nad3 on the accumulation of respiratory chain complexes, the concentration of mitochondrial complex-related proteins, including NADH dehydrogenase subunit 9 (Nad9), CytC (cytochrome c), cytochrome oxidase subunit 2 (Cox 2 ), and $\alpha$ subunit of ATPase ( $\alpha$ ATPase), was detected via immunoblotting. The concentration of complex I subunit Nad9 in dek504 was decreased to 50\% of that in WT, whereas the concentration of proteins forming complexes III and V was increased (32\%, 
$63 \%$, and 70\% increase for Cytc, Cox2, and ATPase-B, respectively) in dek504 (Figure 6c). Therefore, in dek504, the structural components and activity of complex I were downregulated, but those of the other complexes were upregulated, due perhaps to a compensatory metabolic pathway.

\subsection{Mitochondria Morphology Was Affected and an Alternative Respiratory Pathway Was Activated in the dek504-ref Mutant}

Abnormalities in any mitochondrial process can lead to mitochondrial dysfunction, such as mtROS generation and apoptosis [45]. Transmission electron microscopy (TEM) indicated that in WT, the mitochondria formed a continuous closed surface with complex morphology and cristae with densely folded corrugated inner membrane. In contrast, the internal structure of mitochondria in dek504-ref was unclear, lacking the typical cristae, and the mitochondrial matrix was lighter (Figure 7a-d). These results indicate that Dek504 is necessary for the establishment of a normal mitochondrial structure.
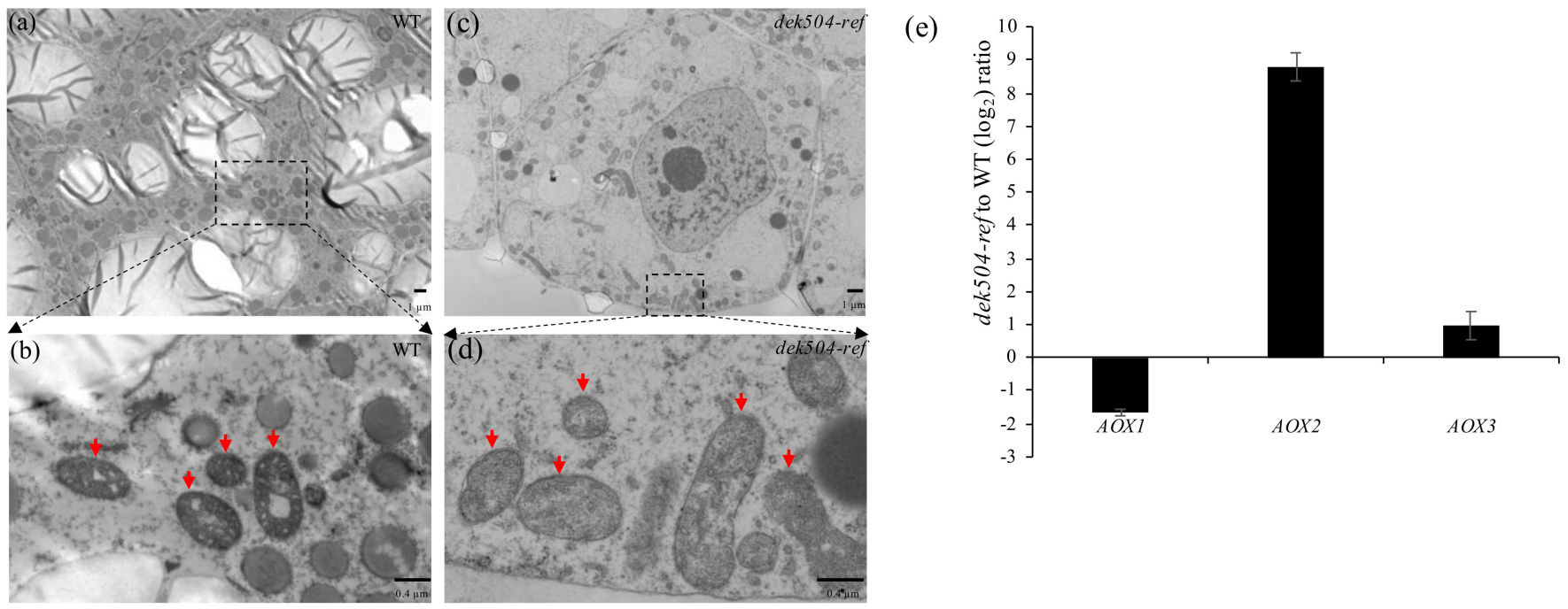

Figure 7. Mitochondrial morphology and AOX expression in the dek504-ref mutant. (a-d) Ultrastructure of developing endosperm in wildtype (WT) (a, b) and dek504-ref (c,d) kernels at 14 days after pollination (DAP). (a,c) Bar $=1 \mu \mathrm{m}$. (b,d) Bar $=0.4 \mu \mathrm{m}$. Red arrows indicate the mitochondria. (e) Quantitative real-time polymerase chain reaction (qRT-PCR) analysis of $A O X 1, A O X 2$, and AOX3 expression in WT and dek504-ref mutant kernels. ZmActin was used as the internal control. Values are presented as means $\pm \mathrm{SE}$ of three biological replicates. $A O X$, alternative oxidase.

Alternative oxidase (AOX) can be induced as an alternative electron transport pathway when the primary respiratory cytochrome c pathway is impaired [46,47]. qRT-PCR was used to evaluate the expression of AOX genes AOX1 (Zm00001d017727), AOX2 (Zm00001d002436), and AOX3 (Zm00001d002434) in dek504. The transcript levels of AOX2 were markedly increased in dek504-ref compared with those in WT (Figure 7e). Consistently, AOX2 protein expression was dramatically increased in dek504-ref (Figure 6c). Therefore, the loss-of-function of DEK504 suppressed complex I activity, subsequently impairing the mitochondrial respiratory pathway and inducing an alternative respiratory pathway in dek504-ref.

\section{Discussion}

\subsection{DEK504 Encodes an E+-Type PPR Protein Involved in Editing at nad3-44 and nad3-317}

Previous studies have shown that PPR proteins serve vital roles in post-transcriptional processes, including RNA editing, splicing, cleavage, and translation in the mitochondria and chloroplasts [14]. There are 49 E+-type PPR genes in maize, of which four genes, namely Dek36, Emp9, Dek40, and Smk6, have been previously characterized [37]. All these 
genes are involved in kernel development, and the loss function of these genes resulted in different phenotypes, with empty pericarp for Emp9, defective kernel for Dek36 and Dek40, and small kernel for Smk6. In the present study, we characterized another E+-type PPR protein, DEK504, which is involved in RNA editing in maize mitochondria. The loss of function of DEK504 resulted in the dek phenotype of the kernel. Although all five known E+-type PPR proteins exhibit C-to-U RNA editing of mitochondrial transcripts, they differ in terms of the editing genes and sites: Dek36 at atp4-59, ccmFN-302, and nad7383; Dek40 at nad5-1916, nad2-26, and cox3-314; Emp9 at ccmB-43 and rps4-335; Smk6 at nad1-740, nad4L-110, nad7-739, mttB-138, and mttB-139; and Dek504 at nad3-44 and nad3$317[37,41,48,49]$. Therefore, the present study unveiled a novel mitochondrion-localized E+-type PPR protein (Figure 4c) involved in C-to-U RNA editing at two novel sites, namely nad3-44 and nad3-317 (Figure 5a).

Previous studies have shown that some E- and DYW-subgroup PPR proteins can interact with the MORF/RIP family proteins to realize their editing functions [9,26-29]. However, the interaction between the E+-PPR protein and other editing factors in maize has not been suggested. Here, we applied a yeast two-hybrid (Y2H) assay to detect the interactions of DEK504 with eight MORF and four organelle RNA recognition motif (ORRM)-containing proteins; however, DEK504 did not interact with any ZmMORF and ZmORRM protein (Supplementary Figure S6, Table S2).

\subsection{Dek504 Editing Sites Are Important for Mitochondrial Function}

Mitochondrial RNA editing has been documented in all major groups of land plants, except bryophytes. In the present study, Dek504 was shown to be essential for C-to-U editing at nad3-44 and nad3-317. gDNA and cDNA sequence analysis revealed that both editing sites were conserved in monocots, with the exception of nad3-317 in rice (Figure 5c). Conserved editing sites among monocot species, including nad7-836 site by SMK1; nad3-61, nad3-62, and cox2-550 by DEK10; nad3-247 and nad3-275 by DEK39; nad7-77, atp1-1292, and atp8-437 by EMP21; and $c c m F_{C}-799$ and nad2-677 by EMP17, have already been revealed previously [12,28,36,50,51]. However, RNA editing at nad3-44 and nad3-317 is not conserved in dicots and can be divided into different types (Figure 5c). Although the conservation of nad3-44 and nad3-317 editing sites differs between monocots and dicots, Leu and Phe were encoded at NAD3-15 and NAD3-106, respectively, and conserved across bryophytes, dicots, and monocots, implying that these residues are important for the functional integrity of the NAD3 protein.

In plants, respiratory metabolism occurs via mitochondrial ETC (mETC), which involves four multisubunit complexes (I-IV) and cytochrome c [34]. Nad3 encodes subunit III of NADH dehydrogenase as part of complex I, which is the primary entry point for electrons into the mETC [52]. The lack of editing at nad3-44 and nad3-317 as a result of the dek504 mutation resulted in the surrogate mutations of Leu to Pro-15 and Phe to Ser-106, respectively. Transmembrane helix prediction indicated that ZmNAD3 possesses three transmembrane helices, with Leu-15 and Phe-106 being located in TMH1 and TMH3, respectively (Supplementary Figure S7).

Mitochondrial Nad3 transcripts of maize contain 66 C-to-U editing sites [53], and only two E-subclass PPR proteins and one DYW-PPR protein have been identified to be involved in RNA editing at these four sites. The Dek10 mutation impeded C-to-U editing at nad3-61 and nad3-62, resulting in an amino acid substitution from Leu to Pro-21, which is conserved among monocots and located in TMH1 [37]. Loss of Dek39 function affected C-to-U editing at nad3-247 and nad3-275, resulting in a conversion from Ser to Pro-83 and Phe to Ser-92, which are located in TMH2 and TMH3, respectively [51]. EMP21 is required for editing at multiple sites, including nad3-275, which is also edited by Dek39 [28,51]. These results indicate that the above five surrogate mutations may negatively impact the structural stability of NAD3 TMH, thus affecting NAD3 function. Taken together, these results indicate that C-to-U editing at nad3-44 and nad3-317 sites is important for NAD3 function. 
Abnormal C-to-U editing in Nad3 transcripts affected the assembly and activity of mitochondrial complex I. Disrupted functions of complex I due to the lack of RNA editing at nad3-250 (slg1) [54], nad3-155, nad3-172, nad3-173, nad3-190, nad3-191 (pps1) [55], nad3-61, nad3-62 (dek10) [36], nad3-247, and nad3-275 (dek39) [51] have been detected. Our results showed that the NADH dehydrogenase activity of complex I in dek504 was reduced to $57 \%$ of that in WT due to the disruption of editing at nad3-44 and nad3-317, which impaired mitochondrial electron transport and structural integrity (Figure 7a-d). The status of mitochondrial electron transport is a vital factor determining plant growth [56,57], and an alternative respiration pathway is activated to compensate for ATP deficiency when the canonical mitochondrial ETC is impaired [58]. In the present study, the transcript levels of $A O X$ genes, particularly $A O X 2$, were noticeably increased in dek504, indicating the activation of the alternative respiratory pathway. Thus, energy shortage may be the major cause of growth defects in dek504.

\section{Materials and Methods}

\subsection{Plant Material and Growth Conditions}

Mutants dek504-ref and g3-mut were derived from the inbred lines $14 \mathrm{~d} 3147$ and 14d3010, respectively. The original name of the allele mutant g4-ems was EMS4-0a0e7, which was purchased from a maize EMS-induced mutant database (http:/ / www.elabcaas. $\mathrm{cn} / \mathrm{memd} /$, accessed on 14 September 2019) [59]. The F2 population of 6192 mutant kernels was used to map the dek504 locus in the B73 background. The maize plants were grown at Shunyi, experimental station of Institute of Crop Sciences, Beijing, China $\left(116.60^{\circ} \mathrm{E}, 40.22^{\circ} \mathrm{N}\right)$ and Nanbin, the experimental station of the Institute of Crop Sciences, Sanya, Hainan Province, China $\left(109.18^{\circ} \mathrm{E}, 18.36^{\circ} \mathrm{N}\right)$ under natural conditions. Arabidopsis (Columbia-0, Col-0) was grown at $22^{\circ} \mathrm{C}$ under a $16 \mathrm{~h} / 8 \mathrm{~h}$ light/dark cycle.

\subsection{Light Microscopy of Cytological Sections, TEM, and SEM}

The dek504-ref and WT kernels from a segregating F2 ear at 12, 15, and 18 DAP were prepared for cytological analysis according to previously published methods [60]. Paraffinembedded sections were stained with toluidine blue and observed under the Nikon Ti microscope (Nikon, Tokyo, Japan). Immature WT and dek504-ref kernels at 15 DAP were treated for TEM. Their dry kernels were sectioned along the embryo, and SEM was used to observe the protein bodies, as described previously [61].

\subsection{Protein and Starch Content Measurement}

The endosperm was separated from the embryo and pericarp, dried to a constant weight, and pulverized with a cryogenic grinder. Protein content was measured according to a previously described protocol [62]. Starch was extracted and measured in $500 \mathrm{mg}$ of powdered sample using an amyloglucosidase/a-amylase starch assay kit (Megazyme, Bray, Ireland), as described previously [63]. Three biological replicates were set for subsequent analyses.

\subsection{Map-Based dek504 Cloning}

Thirty mutant and normal individuals from the same ear of the $F_{2}$ population were collected at 15 DAP and pooled for BSR-Seq analysis according to a published method [64]. An $F_{2}$ population of 6192 mutant kernels was used for fine mapping with the molecular markers listed in Table S2. Following gene annotation and resequencing, the corresponding DNA fragments of the candidate genes in the identified region in dek504-ref and WT-ref were amplified using KOD PLUS DNA polymerase (Toyobo, Osaka, Japan) and sequenced.

\subsection{3'-RACE}

The full-length $3^{\prime}$-termini of the WT and dek504-ref transcripts were isolated using the SMARTerTM RACE cDNA Amplification Kit (Clontech, Mountain View, CA, USA) in accordance with the instructions of the manufacturer using the primers listed in Table 
S2. The amplicons were cloned into the pEASY ${ }^{\circledR}$-Blunt Simple Cloning Vector (TransGen, Beijing, China) and sequenced.

\subsection{Subcellular Localization}

The ORF of Dek504 without the stop codon was amplified from the maize inbred line B73 and cloned into the expression vector pEarleyGate101 to construct the p35S::DEK504eYFP plasmid using the gateway technology (Invitrogen, Carlsbad, CA, USA). This vector was transformed into Arabidopsis thaliana Col-0 using the floral dip method [65], and the lateral root hairs of the transgenic plants were used for YFP observation. MitoTracker Red (Invitrogen) was used to label the mitochondria. Fluorescent signals were observed under a confocal laser scanning microscope (Zeiss LSM700; Carl Zeiss, Oberkochen, Germany) using eYFP (514 $\mathrm{nm}$ excitation and 519-559 $\mathrm{nm}$ emission wavelength), mCherry (587 nm excitation and 590-630 nm emission wavelength), and Mito Tracker Red (581 nm excitation and $644 \mathrm{~nm}$ emission wavelength).

\subsection{RNA Extraction, RT-PCR, and $q R T-P C R$}

RNA was extracted using a commercial kit (Tiangen, Beijing, China), and residual gDNA was removed with RNAse-free DNAse I (NEB) treatment. Then, RNA was reverse transcribed into cDNA using the TransScript II One-Step gDNA Removal Kit and cDNA Synthesis SuperMix Kit (TransGen, Beijing, China) with Oligo (dT) primers. The primers used for RT-PCR and qRT-PCR are listed in Table S2. Glyceraldehyde-3-phosphate dehydrogenase (GAPDH) was used as the internal control.

\subsection{Mitochondrial RNA Editing Analysis}

For RNA editing analysis, 30 seeds of dek504 and WT (following pericarp removal) at 15 DAP were pooled for RNA-Seq, with three biological replicates. Total RNA from each sample was extracted using RNAprep Pure Plant Kit (DP432, Tiangen, Beijing, China) following the manufacturer's protocol. The RNA integrity number and concentration were determined using the Agilent 2100 Bioanalyzer (Agilent, Santa Clara, CA, USA). RNA-Seq libraries were prepared using the Illumina Standard mRNA-Seq Library Preparation Kit (Illumina, San Diego, CA, USA) and sequenced to generate $150 \mathrm{bp}$ paired-end reads on the Illumina HiSeq4000 (Illumina) platform. Adaptor sequences, unknown nucleotides $>5 \%$, or percentage of reads with sequencing error rates $<1 \%$ were removed using a Perl script. Each sample of the clean reads with two biological replicates was first merged and aligned to the B73 reference genome (AGPv4) using HISAT2 (v2.1.0) [66]. Single-nucleotide polymorphic sites were identified by Samtools (v1.3.1) [67]. Editing frequencies of the same sites were calculated using Samtools mpileup (https:/ / www.htslib.org, accessed on 4 May 2019). Sites covering over 10 reads were used to analyze the editing efficiency. Sites located within the gene-coding region were selected to analyze the changes in editing efficiency.

To analyze the editing efficiency at nad3-44 and nad3-317, mitochondrial cDNA was amplified, and the PCR products were cloned into the $p E A S Y{ }^{\circledR}$-Blunt Simple Cloning Vector (TransGen, Beijing, China). Ninety clones each from WT and dek504-ref PCR products were separately sequenced. The primers used are listed in Table S2.

To exclude possible polymorphisms (SNPs) at the nad3-44 and nad3-317 sites in the dek504 mutant and WT mitochondrial genomes, DNA of 10 kernels were extracted from dek504, WT-ref, and B73 each. A 357 bp DNA fragment of the nad3 region was amplified using specific primers (nad3-F and nad3-R). DNA fragments were sequenced separately for each sample by ABI 3730XL sequencer.

\subsection{Isolation and Analysis of Mitochondria Complexes and Western Blotting}

Crude mitochondria were extracted from 15 DAP kernels of dek504-ref and WT (without pericarp) according to a previously described method [64]. For BN-PAGE and NADH dehydrogenase activity of complex I analysis, mitochondrial proteins $(100 \mu \mathrm{g})$ were solubi- 
lized using the NativePAGETM Sample Prep Kit (Invitrogen, Carlsbad, CA), as described previously [64].

Mitochondrial proteins $(20 \mu \mathrm{g})$ extracted from WT and dek504-ref kernels at 15 DAP were used for Western blotting. Proteins were separated by SDS-PAGE, transferred to a polyvinylidene difluoride (PVDF) membrane, and incubated overnight at $4{ }^{\circ} \mathrm{C}$ with various target protein antibodies. Following incubation with the HRP-conjugated secondary antibody, the signals were visualized using the SuperSignalTM West Pico PLUS Chemiluminescent Substrate Kit (Thermo, Waltham, MA, USA) in accordance with the instructions of the manufacturer. The antibody dilutions used were 1:1000 for Cox2 (Agrisera), 1:1000 for NAD9 (PHYTOAB), 1:2000 for Cyt-c (Agrisera), 1:2000 for AOX (PHYTOAB), and 1:1000 for ATPase-B (Agrisera).

Supplementary Materials: The following supporting information can be downloaded at: https: //www.mdpi.com/article/10.3390/ijms23052513/s1.

Author Contributions: Y.C. and J.W. conceived and designed the experiments; Z.W. and Y.C. performed the experiments and analyzed the data; W.C., S.Z., R.C. and R.G. provided technical assistance on the experiments; J.L. and J.F. completed RNA sequencing data analysis; G.W. provided funding support; Y.C. and Z.W. wrote the manuscript; R.G reviewed and edited the manuscript. All authors have read and agreed to the published version of the manuscript.

Funding: This work was funded by the National Natural Science Foundation of China (31901563), the Agricultural Science and Technology Innovation Program of the Chinese Academy of Agricultural Sciences (ASTIP, CAAS), and the China Agriculture Research System (CARS-02-13).

Institutional Review Board Statement: Not applicable.

Informed Consent Statement: Not applicable.

Data Availability Statement: All of the data generated or analyzed during this study are included in the published article.

Conflicts of Interest: The authors declare that they have no known competing financial interest or personal relationship that could have appeared to influence the work reported in this paper.

\section{References}

1. Takenaka, M.; Zehrmann, A.; Verbitskiy, D.; Hartel, B.; Brennicke, A. RNA editing in plants and its evolution. Annu. Rev. Genet. 2013, 47, 335-352. [CrossRef] [PubMed]

2. Shikanai, T. RNA editing in plants: Machinery and flexibility of site recognition. Biochim. Biophys. Acta 2015, 1847, 779-785. [CrossRef] [PubMed]

3. Ichinose, M.; Sugita, M. RNA Editing and Its Molecular Mechanism in Plant Organelles. Genes 2016, 8, 5. [CrossRef] [PubMed]

4. Maier, U.G.; Bozarth, A.; Funk, H.T.; Zauner, S.; Rensing, S.A.; Schmitz-Linneweber, C.; Borner, T.; Tillich, M. Complex chloroplast RNA metabolism: Just debugging the genetic programme? BMC Biol. 2008, 6, 36. [CrossRef]

5. Chateigner-Boutin, A.L.; Small, I. Plant RNA editing. RNA Biol. 2010, 7, 213-219. [CrossRef]

6. Covello, P.S.; Gray, M.W. RNA editing in plant mitochondria. Nature 1989, 341, 662-666. [CrossRef]

7. Hiesel, R.; Wissinger, B.; Schuster, W.; Brennicke, A. RNA editing in plant mitochondria. Science 1989, 246, 1632-1634. [CrossRef]

8. Kotera, E.; Tasaka, M.; Shikanai, T. A pentatricopeptide repeat protein is essential for RNA editing in chloroplasts. Nature 2005, 433, 326-330. [CrossRef]

9. Dai, D.; Jin, L.; Huo, Z.; Yan, S.; Ma, Z.; Qi, W.; Song, R. Maize pentatricopeptide repeat protein DEK53 is required for mitochondrial RNA editing at multiple sites and seed development. J. Exp. Bot. 2020, 71, 6246-6261. [CrossRef]

10. Liu, X.Y.; Jiang, R.C.; Wang, Y.; Tang, J.J.; Sun, F.; Yang, Y.Z.; Tan, B.C. ZmPPR26, a DYW-type pentatricopeptide repeat protein, is required for C-to-U RNA editing at atpA-1148 in maize chloroplasts. J. Exp. Bot. 2021, 72, 4809-4821. [CrossRef]

11. Malbert, B.; Burger, M.; Lopez-Obando, M.; Baudry, K.; Launay-Avon, A.; Hartel, B.; Verbitskiy, D.; Jorg, A.; Berthome, R.; Lurin C.; et al. The Analysis of the Editing Defects in the dyw2 Mutant Provides New Clues for the Prediction of RNA Targets of Arabidopsis E+-Class PPR Proteins. Plants 2020, 9, 280. [CrossRef]

12. Wang, Y.; Liu, X.Y.; Huang, Z.Q.; Li, Y.Y.; Yang, Y.Z.; Sayyed, A.; Sun, F.; Gu, Z.Q.; Wang, X.; Tan, B.C. PPR-DYW Protein EMP17 Is Required for Mitochondrial RNA Editing, Complex III Biogenesis, and Seed Development in Maize. Front. Plant Sci. 2021, 12, 693272. [CrossRef] 
13. Wang, Y.; Wang, Y.; Ren, Y.; Duan, E.; Zhu, X.; Hao, Y.; Zhu, J.; Chen, R.; Lei, J.; Teng, X.; et al. white panicle2 encoding thioredoxin $\mathrm{z}$, regulates plastid RNA editing by interacting with multiple organellar RNA editing factors in rice. New Phytol. 2021, 229, 2693-2706. [CrossRef]

14. Schmitz-Linneweber, C.; Small, I. Pentatricopeptide repeat proteins: A socket set for organelle gene expression. Trends Plant Sci. 2008, 13, 663-670. [CrossRef]

15. Takenaka, M.; Verbitskiy, D.; Zehrmann, A.; Hartel, B.; Bayer-Csaszar, E.; Glass, F.; Brennicke, A. RNA editing in plant mitochondria-connecting RNA target sequences and acting proteins. Mitochondrion 2014, 19 Pt B, 191-197. [CrossRef]

16. Lurin, C.; Andres, C.; Aubourg, S.; Bellaoui, M.; Bitton, F.; Bruyere, C.; Caboche, M.; Debast, C.; Gualberto, J.; Hoffmann, B.; et al. Genome-wide analysis of Arabidopsis pentatricopeptide repeat proteins reveals their essential role in organelle biogenesis. Plant Cell 2004, 16, 2089-2103. [CrossRef]

17. Cheng, S.; Gutmann, B.; Zhong, X.; Ye, Y.; Fisher, M.F.; Bai, F.; Castleden, I.; Song, Y.; Song, B.; Huang, J.; et al. Redefining the structural motifs that determine RNA binding and RNA editing by pentatricopeptide repeat proteins in land plants. Plant J. 2016, 85, 532-547. [CrossRef]

18. Barkan, A.; Small, I. Pentatricopeptide repeat proteins in plants. Annu. Rev. Plant Biol. 2014, 65, 415-442. [CrossRef]

19. Bentolila, S.; Heller, W.P.; Sun, T.; Babina, A.M.; Friso, G.; van Wijk, K.J.; Hanson, M.R. RIP1, a member of an Arabidopsis protein family, interacts with the protein RARE1 and broadly affects RNA editing. Proc. Natl. Acad. Sci. USA 2012, 109, E1453-E1461. [CrossRef]

20. Takenaka, M.; Zehrmann, A.; Verbitskiy, D.; Kugelmann, M.; Hartel, B.; Brennicke, A. Multiple organellar RNA editing factor (MORF) family proteins are required for RNA editing in mitochondria and plastids of plants. Proc. Natl. Acad. Sci. USA 2012, 109, 5104-5109. [CrossRef]

21. Sun, T.; Germain, A.; Giloteaux, L.; Hammani, K.; Barkan, A.; Hanson, M.R.; Bentolila, S. An RNA recognition motif-containing protein is required for plastid RNA editing in Arabidopsis and maize. Proc. Natl. Acad. Sci. USA 2013, 110, E1169-E1178 [CrossRef] [PubMed]

22. Zhang, F.; Tang, W.; Hedtke, B.; Zhong, L.; Liu, L.; Peng, L.; Lu, C.; Grimm, B.; Lin, R. Tetrapyrrole biosynthetic enzyme protoporphyrinogen IX oxidase 1 is required for plastid RNA editing. Proc. Natl. Acad. Sci. USA 2014, 111, 2023-2028. [CrossRef] [PubMed]

23. Sun, T.; Shi, X.; Friso, G.; Van Wijk, K.; Bentolila, S.; Hanson, M.R. A zinc finger motif-containing protein is essential for chloroplast RNA editing. PLoS Genet. 2015, 11, e1005028. [CrossRef] [PubMed]

24. Glass, F.; Hartel, B.; Zehrmann, A.; Verbitskiy, D.; Takenaka, M. MEF13 Requires MORF3 and MORF8 for RNA Editing at Eight Targets in Mitochondrial mRNAs in Arabidopsis thaliana. Mol. Plant 2015, 8, 1466-1477. [CrossRef]

25. Sandoval, R.; Boyd, R.D.; Kiszter, A.N.; Mirzakhanyan, Y.; Santibanez, P.; Gershon, P.D.; Hayes, M.L. Stable native RIP9 complexes associate with C-to-U RNA editing activity, PPRs, RIPs, OZ1, ORRM1 and ISE2. Plant J. 2019, 99, 1116-1126. [CrossRef]

26. Liu, R.; Cao, S.K.; Sayyed, A.; Yang, H.H.; Zhao, J.; Wang, X.; Jia, R.X.; Sun, F.; Tan, B.C. The DYW-subgroup pentatricopeptide repeat protein PPR27 interacts with ZmMORF1 to facilitate mitochondrial RNA editing and seed development in maize. J. Exp. Bot. 2020, 71, 5495-5505. [CrossRef]

27. Ren, R.C.; Yan, X.W.; Zhao, Y.J.; Wei, Y.M.; Lu, X.; Zang, J.; Wu, J.W.; Zheng, G.M.; Ding, X.H.; Zhang, X.S.; et al. The novel E-subgroup pentatricopeptide repeat protein DEK55 is responsible for RNA editing at multiple sites and for the splicing of nad1 and nad4 in maize. BMC Plant Biol. 2020, 20, 553. [CrossRef]

28. Wang, Y.; Liu, X.Y.; Yang, Y.Z.; Huang, J.; Sun, F.; Lin, J.; Gu, Z.Q.; Sayyed, A.; Xu, C.; Tan, B.C. Empty Pericarp21 encodes a novel PPR-DYW protein that is required for mitochondrial RNA editing at multiple sites, complexes I and V biogenesis, and seed development in maize. PLoS Genet. 2019, 15, e1008305. [CrossRef]

29. Liu, Y.J.; Xiu, Z.H.; Meeley, R.; Tan, B.C. Empty pericarp5 encodes a pentatricopeptide repeat protein that is required for mitochondrial RNA editing and seed development in maize. Plant Cell 2013, 25, 868-883. [CrossRef]

30. Clifton, S.W.; Minx, P.; Fauron, C.M.; Gibson, M.; Allen, J.O.; Sun, H.; Thompson, M.; Barbazuk, W.B.; Kanuganti, S.; Tayloe, C.; et al. Sequence and comparative analysis of the maize NB mitochondrial genome. Plant Physiol. 2004, 136, 3486-3503. [CrossRef]

31. Ordog, S.H.; Higgins, V.J.; Vanlerberghe, G.C. Mitochondrial alternative oxidase is not a critical component of plant viral resistance but may play a role in the hypersensitive response. Plant Physiol. 2002, 129, 1858-1865. [CrossRef]

32. Vanlerberghe, G.C. Alternative oxidase: A mitochondrial respiratory pathway to maintain metabolic and signaling homeostasis during abiotic and biotic stress in plants. Int. J. Mol. Sci. 2013, 14, 6805-6847. [CrossRef]

33. Sweetlove, L.J.; Taylor, N.L.; Leaver, C.J. Isolation of intact, functional mitochondria from the model plant Arabidopsis thaliana. Methods Mol. Biol. 2007, 372, 125-136.

34. Dudkina, N.V.; Heinemeyer, J.; Sunderhaus, S.; Boekema, E.J.; Braun, H.P. Respiratory chain supercomplexes in the plant mitochondrial membrane. Trends Plant Sci. 2006, 11, 232-240. [CrossRef]

35. Siedow, J.N.; Day, D. Respiration and photorespiration. In Biochemistry and Molecular Biology of Plants; Buchanan, B.B., Gruissem, W., Jones, R.L., Eds.; American Society of Plant Physiologists: Rockville, MD, USA, 2000; pp. 676-728.

36. Qi, W.; Tian, Z.; Lu, L.; Chen, X.; Chen, X.; Zhang, W.; Song, R. Editing of Mitochondrial Transcripts nad3 and cox2 by Dek10 Is Essential for Mitochondrial Function and Maize Plant Development. Genetics 2017, 205, 1489-1501. [CrossRef] 
37. Wang, G.; Zhong, M.; Shuai, B.; Song, J.; Zhang, J.; Han, L.; Ling, H.; Tang, Y.; Wang, G.; Song, R. E+ subgroup PPR protein defective kernel 36 is required for multiple mitochondrial transcripts editing and seed development in maize and Arabidopsis. New Phytol. 2017, 214, 1563-1578. [CrossRef]

38. Karpenahalli, M.R.; Lupas, A.N.; Soding, J. TPRpred: A tool for prediction of TPR-, PPR- and SEL1-like repeats from protein sequences. BMC Bioinform. 2007, 8, 2. [CrossRef]

39. Nelson, B.K.; Cai, X.; Nebenfuhr, A. A multicolored set of in vivo organelle markers for co-localization studies in Arabidopsis and other plants. Plant J. 2007, 51, 1126-1136. [CrossRef]

40. Barkan, A.; Rojas, M.; Fujii, S.; Yap, A.; Chong, Y.S.; Bond, C.S.; Small, I. A combinatorial amino acid code for RNA recognition by pentatricopeptide repeat proteins. PLoS Genet. 2012, 8, e1002910. [CrossRef]

41. Yang, Y.Z.; Ding, S.; Wang, H.C.; Sun, F.; Huang, W.L.; Song, S.; Xu, C.; Tan, B.C. The pentatricopeptide repeat protein EMP9 is required for mitochondrial $\mathrm{ccmB}$ and rps4 transcript editing, mitochondrial complex biogenesis and seed development in maize. New Phytol. 2017, 214, 782-795. [CrossRef]

42. Sun, F.; Zhang, X.; Shen, Y.; Wang, H.; Liu, R.; Wang, X.; Gao, D.; Yang, Y.Z.; Liu, Y.; Tan, B.C. The pentatricopeptide repeat protein EMPTY PERICARP8 is required for the splicing of three mitochondrial introns and seed development in maize. Plant J. 2018, 95, 919-932. [CrossRef]

43. Li, X.L.; Huang, W.L.; Yang, H.H.; Jiang, R.C.; Sun, F.; Wang, H.C.; Zhao, J.; Xu, C.H.; Tan, B.C. EMP18 functions in mitochondrial atp6 and cox2 transcript editing and is essential to seed development in maize. New Phytol. 2019, 221, 896-907. [CrossRef]

44. Sage, D.; Unser, M. Teaching image-processing programming in Java. IEEE Signal Process. Mag. 2003, 20, 43-52. [CrossRef]

45. Tal, M.C.; Sasai, M.; Lee, H.K.; Yordy, B.; Shadel, G.S.; Iwasaki, A. Absence of autophagy results in reactive oxygen speciesdependent amplification of RLR signaling. Proc. Natl. Acad. Sci. USA 2009, 106, 2770-2775. [CrossRef]

46. Kuhn, K.; Yin, G.; Duncan, O.; Law, S.R.; Kubiszewski-Jakubiak, S.; Kaur, P.; Meyer, E.; Wang, Y.; Small, C.C.; Giraud, E.; et al Decreasing electron flux through the cytochrome and/or alternative respiratory pathways triggers common and distinct cellular responses dependent on growth conditions. Plant Physiol. 2015, 167, 228-250. [CrossRef]

47. Vanlerberghe, G.C.; Martyn, G.D.; Dahal, K. Alternative oxidase: A respiratory electron transport chain pathway essential for maintaining photosynthetic performance during drought stress. Physiol. Plant 2016, 157, 322-337. [CrossRef]

48. Ren, R.C.; Lu, X.; Zhao, Y.J.; Wei, Y.M.; Wang, L.L.; Zhang, L.; Zhang, W.T.; Zhang, C.; Zhang, X.S.; Zhao, X.Y. Pentatricopeptide repeat protein DEK40 is required for mitochondrial function and kernel development in maize. J. Exp. Bot. 2019, 70, 6163-6179. [CrossRef]

49. Ding, S.; Liu, X.Y.; Wang, H.C.; Wang, Y.; Tang, J.J.; Yang, Y.Z.; Tan, B.C. SMK6 mediates the C-to-U editing at multiple sites in maize mitochondria. J. Plant Physiol. 2019, 240, 152992. [CrossRef]

50. Li, X.J.; Zhang, Y.F.; Hou, M.; Sun, F.; Shen, Y.; Xiu, Z.H.; Wang, X.; Chen, Z.L.; Sun, S.S.; Small, I.; et al. Small kernel 1 encodes a pentatricopeptide repeat protein required for mitochondrial nad7 transcript editing and seed development in maize (Zea mays) and rice (Oryza sativa). Plant J. 2014, 79, 797-809. [CrossRef]

51. Li, X.; Gu, W.; Sun, S.; Chen, Z.; Chen, J.; Song, W.; Zhao, H.; Lai, J. Defective Kernel 39 encodes a PPR protein required for seed development in maize. J. Integr. Plant Biol. 2018, 60, 45-64. [CrossRef] [PubMed]

52. Braun, H.P.; Binder, S.; Brennicke, A.; Eubel, H.; Fernie, A.R.; Finkemeier, I.; Klodmann, J.; Konig, A.C.; Kuhn, K.; Meyer, E.; et al The life of plant mitochondrial complex I. Mitochondrion 2014, 19 Pt B, 295-313. [CrossRef]

53. Li, M.; Xia, L.; Zhang, Y.; Niu, G.; Li, M.; Wang, P.; Zhang, Y.; Sang, J.; Zou, D.; Hu, S.; et al. Plant editosome database: A curated database of RNA editosome in plants. Nucleic Acids Res. 2019, 47, D170-D174. [CrossRef] [PubMed]

54. Yuan, H.; Liu, D. Functional disruption of the pentatricopeptide protein SLG1 affects mitochondrial RNA editing, plant development, and responses to abiotic stresses in Arabidopsis. Plant J. 2012, 70, 432-444. [CrossRef] [PubMed]

55. Xiao, H.; Zhang, Q.; Qin, X.; Xu, Y.; Ni, C.; Huang, J.; Zhu, L.; Zhong, F.; Liu, W.; Yao, G.; et al. Rice PPS1 encodes a DYW motif-containing pentatricopeptide repeat protein required for five consecutive RNA-editing sites of nad3 in mitochondria. New Phytol. 2018, 220, 878-892. [CrossRef] [PubMed]

56. Stitt, M. Limitation of Photosynthesis by Carbon Metabolism: I. Evidence for Excess Electron Transport Capacity in Leaves Carrying Out Photosynthesis in Saturating Light and $\mathrm{CO}_{2}$. Plant Physiol. 1986, 81, 1115-1122. [CrossRef] [PubMed]

57. Griffin, K.L.; Anderson, O.R.; Gastrich, M.D.; Lewis, J.D.; Lin, G.; Schuster, W.; Seemann, J.R.; Tissue, D.T.; Turnbull, M.H.; Whitehead, D. Plant growth in elevated $\mathrm{CO}_{2}$ alters mitochondrial number and chloroplast fine structure. Proc. Natl. Acad. Sci. USA 2001, 98, 2473-2478. [CrossRef] [PubMed]

58. Juszczuk, I.M.; Rychter, A.M. Alternative oxidase in higher plants. Acta Biochim. Pol. 2003, 50, 1257-1271. [CrossRef]

59. Lu, X.; Liu, J.; Ren, W.; Yang, Q.; Chai, Z.; Chen, R.; Wang, L.; Zhao, J.; Lang, Z.; Wang, H.; et al. Gene-Indexed Mutations in Maize. Mol. Plant 2018, 11, 496-504. [CrossRef]

60. He, Y.; Wang, J.; Qi, W.; Song, R. Maize Dek15 Encodes the Cohesin-Loading Complex Subunit SCC4 and Is Essential for Chromosome Segregation and Kernel Development. Plant Cell 2019, 31, 465-485. [CrossRef]

61. Lending, C.; Larkins, B. Effect of thefloury-2 locus on protein body formation during maize endosperm development. Protoplasma 1992, 171, 123-133. [CrossRef]

62. Wang, G.; Sun, X.; Wang, G.; Wang, F.; Gao, Q.; Sun, X.; Tang, Y.; Chang, C.; Lai, J.; Zhu, L.; et al. Opaque7 encodes an acyl-activating enzyme-like protein that affects storage protein synthesis in maize endosperm. Genetics 2011, 189, 1281-1295. [CrossRef] 
63. Wang, G.; Qi, W.; Wu, Q.; Yao, D.; Zhang, J.; Zhu, J.; Wang, G.; Wang, G.; Tang, Y.; Song, R. Identification and Characterization of Maize floury4 as a Novel Semidominant Opaque Mutant That Disrupts Protein Body Assembly. Plant Physiol. 2014, 165, 582-594. [CrossRef]

64. Chen, W.; Cui, Y.; Wang, Z.; Chen, R.; He, C.; Liu, Y.; Du, X.; Liu, Y.; Fu, J.; Wang, G.; et al. Nuclear-Encoded Maturase Protein 3 Is Required for the Splicing of Various Group II Introns in Mitochondria during Maize (Zea mays L.) Seed Development. Plant Cell Physiol. 2021, 62, 293-305. [CrossRef]

65. Clough, S.J.; Bent, A.F. Floral dip: A simplified method for Agrobacterium-mediated transformation of Arabidopsis thaliana. Plant J. 1998, 16, 735-743. [CrossRef]

66. Kim, D.; Paggi, J.M.; Park, C.; Bennett, C.; Salzberg, S.L. Graph-based genome alignment and genotyping with HISAT2 and HISAT-genotype. Nat. Biotechnol. 2019, 37, 907-915. [CrossRef]

67. Li, H.; Handsaker, B.; Wysoker, A.; Fennell, T.; Ruan, J.; Homer, N.; Marth, G.; Abecasis, G.; Durbin, R.; Genome Project Data Processing, S. The Sequence Alignment/Map format and SAMtools. Bioinformatics 2009, 25, 2078-2079. [CrossRef] 